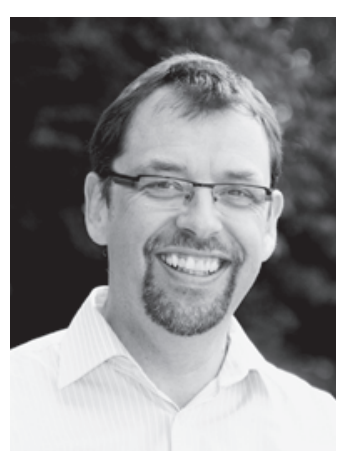

\title{
Latest Publications You Should Not Miss
}

\author{
Selected by \\ Rainer Lüdtke \\ Essen
}

Wildt S, Nordgaard I, Hansen U, Brockmann E, Rumessen JJ: A randomised double-blind placebocontrolled trial with Lactobacillus acidophilus La-5 and Bifidobacterium animalis subsp. lactis BB-12 for maintenance of remission in ulcerative colitis. J Crohns Colitis 2011;5:115-121.

Background and Aims: To investigate the clinical effect of treatment with Lactobacillus acidophilus La-5 and Bifidobacterium animalis subsp. lactis BB-12 (Probio-Tec AB-25) to maintain remission in patients with ulcerative colitis.

Methods: Patients with left-sided ulcerative colitis in remission - including proctitis and at least one relapse within the last year were randomised (2:1) in a double-blind placebo-controlled study to Probio-Tec AB-25 or placebo for 52 weeks. The patients were evaluated clinically, endoscopically and histologically at entry and if relapsing. No other medication for ulcerative colitis than the study drug was allowed during the study. Primary endpoint was maintenance of clinical remission, secondary endpoints were comparisons of days to relapse, and safety and tolerability of the study drug. The concentrations of the probiotic bacterial strains in stool were analysed in a subset of patients.

Results: Thirty-two patients were randomised. Twenty patients received Probio-Tec AB-25 and twelve patients received placebo. Five patients $(25 \%)$ in the Probio-Tec AB-25 group and one patient $(8 \%)$ in the placebo group maintained remission after 1 year of treatment $(\mathrm{p}=0.37)$. The median time to relapse was 125.5 days (range 11-391 days) in the probiotic group and 104 days (range 28-369 days) in the placebo group respectively ( $\mathrm{p}=0.683$ ). Probio-Tec AB-25 was overall well tolerated.

Conclusions: In this small randomised placebo-controlled trial no significant clinical benefit of Probio-Tec AB-25 could be demonstrated in comparison with placebo for maintaining remission in patients with left-sided ulcerative colitis. A difference may be achieved in larger studies, but the clinical significance of this would be questionable.

\section{Cao H, Liu J, Zhu C: Wet cupping therapy for treatment of herpes zoster: a systematic review of randomized controlled trials. Altern Ther 2010;1: 48-54.}

Background: Wet cupping is a traditional Chinese medicine therapy commonly used in treating herpes zoster in China, and clinical studies have shown that wet cupping may have beneficial effect on herpes zoster compared with Western medication.

Methods: We included randomized controlled trials (RCTs) on wet cupping for herpes zoster. We searched PubMed, the Cochrane Library (Issue 3, 2008), China Network Knowledge Infrastructure (CNKI), Chinese Scientific Journal Database (VIP), and Wan Fang Database. All searches ended in February 2009. Two authors extracted data and assessed the trials quality independently. RevMan 5.0.18 software (the Cochrane Collaboration, the Nordic Cochrane Centre, Copenhagen, Denmark) was used for data analysis with effect estimate presented as relative risk (RR) and mean difference (MD) with a 95\% confidence interval (CI).

Results: Eight RCTs involving 651 patients were included, and the methodological quality of trials was generally fair in terms of randomization, blinding, and intention-to-treat analysis. Meta-analyses showed wet cupping was superior to medication in the number of cured patients (RR $2.49,95 \%$ CI 1.91 to 3.24 ; $\mathrm{P}<.00001$ ), the number of patients with improved symptoms ( $\mathrm{RR} 1.15,95 \%$ CI 1.05 to $1.26, \mathrm{P}=.003$ ), and reducing the incidence rate of postherpetic neuralgia (RR $0.06,95 \%$ CI 0.02 to $0.25, \mathrm{P}=.0001)$. Wet cupping plus medication was significantly better than medication alone on number of cured patients (RR 1.93, 95\% CI 1.23 to $3.04, \mathrm{P}=.005)$ but demonstrated no difference in symptom improvement ( $\mathrm{RR} 1.00,95 \% \mathrm{CI} 0.92$ to $1.08, \mathrm{P}=.98$ ). There were no serious adverse effects related to wet cupping therapy in the included trials.

Conclusion: Wet cupping appears to be effective in the treatment of herpes zoster. However, further large, rigorously designed trials are warranted.

\begin{tabular}{ll}
\hline KARGER & @ 2011 S. Karger GmbH, Freiburg \\
Fax +497614520714 & Accessible online at: \\
$\begin{array}{l}\text { Information@Karger.de } \\
\text { www.karger.com }\end{array}$ & www.karger.com/fok
\end{tabular}


Sánchez-Villegas A, Verberne L, De Irala J, RuízCanela M, Toledo E, Serra-Majem L, Martínez-González MA: Dietary fat intake and the risk of depression: the SUN Project. PLoS One 2011;26;6:e16268.

Emerging evidence relates some nutritional factors to depression risk. However, there is a scarcity of longitudinal assessments on this relationship.

Objective: To evaluate the association between fatty acid intake or the use of culinary fats and depression incidence in a Mediterranean population.

Material and Methods: Prospective cohort study (1999-2010) of 12,059 Spanish university graduates (mean age: 37.5 years) initially free of depression with permanently open enrolment. At baseline, a 136-item validated food frequency questionnaire was used to estimate the intake of fatty acids (saturated fatty acids (SFA), polyunsaturated fatty acids (PUFA), trans unsaturated fatty acids (TFA) and monounsaturated fatty acids (MUFA) and culinary fats (olive oil, seed oils, butter and margarine)). During follow-up participants were classified as incident cases of depression if they reported a new clinical diagnosis of depression by a physician and/or initiated the use of antidepressant drugs. Cox regression models were used to calculate Hazard Ratios (HR) of incident depression and their $95 \%$ confidence intervals (CI) for successive quintiles of fats.

Results: During follow-up (median: 6.1 years), 657 new cases of depression were identified. Multivariable-adjusted HR (95\% CI) for depression incidence across successive quintiles of TFA intake were: 1 (ref), 1.08 (0.82-1.43), 1.17 (0.88-1.53), 1.28 (0.97-1.68), 1.42 (1.09-1.84) with a significant dose-response relationship ( $\mathrm{p}$ for trend $=0,003$ ). Results did not substantially change after adjusting for potential lifestyle or dietary confounders, including adherence to a Mediterranean Dietary Pattern. On the other hand, an inverse and significant dose-response relationship was obtained for MUFA ( $\mathrm{p}$ for trend $=0.05$ ) and PUFA ( $\mathrm{p}$ for trend $=0.03$ ) intake

Conclusions: A detrimental relationship was found between TFA intake and depression risk, whereas weak inverse associations were found for MUFA, PUFA and olive oil. These findings suggest that cardiovascular disease and depression may share some common nutritional determinants related to subtypes of fat intake.

\section{Kligler B, Homel P, Blank AE, Kenney J, Levenson H, Merrel W: Randomized trial of the effect of an integrative medicine approach to the management of asthma in adults on disease-related quality of life and pulmonary function. Altern Ther 2011;17:10-15.}

Purpose: The purpose of this study was to test the effectiveness of an integrative medicine approach to the management of asthma compared to standard clinical care on quality of life (QOL) and clinical outcomes.

Methods: This was a prospective parallel group repeated measurement randomized design. Participants were adults aged 18 to 80 years with asthma. The intervention consisted of six group sessions on the use of nutritional manipulation, yoga techniques, and journaling. Participants also received nutritional supplements: fish oil, vitamin C, and a standardized hops extract. The control group received usual care. Primary outcome measures were the Asthma Quality of Life Questionnaire (AQLQ), the Medical Outcomes Study Short Form-12 (SF-12), and standard pulmonary function tests (PFTs).

Results: In total, 154 patients were randomized and included in the intention-to-treat analysis (77 control, 77 treatment). Treatment participants showed greater improvement than controls at 6 months for the AQLQ total score $(\mathrm{P}<.001)$ and for three subscales, Activity $(\mathrm{P}<.001)$, Symptoms $(\mathrm{P}=.02)$, and Emotion $(\mathrm{P}<.001)$. Treatment participants also showed greater improvement than controls on three of the SF-12 subscales: Physical Functioning $(\mathrm{P}=.003)$; Role Limitations, Physical $(\mathrm{P}<.001)$ and Social Functioning $(\mathrm{P}=.03)$, as well as in the aggregate scores for Physical and Mental Health $(\mathrm{P}=.003$ and .02 , respectively). There was no change in PFTs in either group.

Conclusion: A low-cost group-oriented integrative medicine intervention can lead to significant improvement in QOL in adults with asthma.

\section{Di Cesare A, Giombini A, Di Cesare M, Ripani M,} Vulpiani MC, Saraceni VM: Comparison between the effects of trigger point mesotherapy versus acupuncture points mesotherapy in the treatment of chronic low back pain: a short term randomized controlled trial. Complement Ther Med 2011;19:19-26.

Objective: The goal of this study was to compare the effects of trigger point (TRP) mesotherapy and acupuncture (ACP) mesotherapy in the treatment of patients with chronic low back pain.

Design: Short term randomized controlled trial.

Setting: 62 subjects with chronic low back pain were recruited at outpatients Physical Medicine and Rehabilitation Clinic at the University of Rome 'La Sapienza' in the period between July 2006 and May 2008.

Intervention: Study subjects were assigned to receive 4 weeks treatments with either trigger point mesotherapy (TRP mesotherapy, $n=29$ ) or acupoints mesotherapy (ACP mesotherapy, $\mathrm{n}=33$ ).

Main Outcome Measures: Pain intensity with a pain visual analogic scale (VAS) and verbal rating scale (VRS) and pain disability with McGill Pain Questionnaire Short Form (SFMPQ), Roland Morris Disability Questionnaire (RMQ) and Oswestry Low Back Pain Disability Questionaire (ODQ).

Results: ACP mesotherapy shows a more effective results in VRS and VAS measures in the follow-up $(\mathrm{p}(\mathrm{VRS})=<.001$ and $\mathrm{p}(\mathrm{VAS})=$ $<.001)$. The SF-MPQ measure shows a better results for ACP instead of TRP with statistically significant differences between groups and time $(\mathrm{p}=.035)$. Participants reported a slight discomfort at the time of the inoculation, and $15 \%$ reported slight neck pain in ACP mesotherapy group.

Conclusions: Our results suggest that the response to ACP mesotherapy may be greater than the response to TRP mesotherapy in the short term follow-up. This technique could be nevertheless a viable option as an adjunct treatment in an overall treatment planning of CLBP. 